colorectal liver metastasis: results from a Scandinavian multicenter randomized controlled trial (LIGRO Trial). Ann Surg. 2017 [Epub ahead of print].

2. Aloia TA, Vauthey JN. Associating liver partition and portal vein ligation for staged hepatectomy (ALPPS): what is gained and what is lost? Ann Surg. 2012;256:e9. author reply e16-9.

3. Muratore A, Zimmitti G, Ribero D, et al. Chemotherapy between the first and second stages of a two-stage hepatectomy for colorectal liver metastases: should we routinely recommend it? Ann Surg Oncol. 2012;19:1310-1315.

4. Adam R, Imai K, Castro Benitez C, et al. Outcome after associating liver partition and portal vein ligation for staged hepatectomy and conventional two-stage hepatectomy for colorectal liver metastases. Br J Surg. 2016;103:1521-1529.

5. Shindoh J, Truty MJ, Aloia TA, et al. Kinetic growth rate after portal vein embolization predicts posthepatectomy outcomes: toward zero liverrelated mortality in patients with colorectal liver metastases and small future liver remnant. $\mathrm{J} \mathrm{Am}$ Coll Surg. 2013;216:201-209.

\section{Comment on "Prediction} of Hepatocellular Carcinoma Recurrence Beyond Milan Criteria After Resection: Validation of a Clinical Risk Score in Aninternational Cohort"

W

Editors:

e read with great interest the article by Zheng et al, ${ }^{1}$ which validated the ability of a previously reported clinical risk score $^{2}$ in predicting recurrence patterns and incidences after surgical resection of hepatocellular carcinoma (HCC). A creative classification was proposed by the authors to distinguish patients with postoperative $\mathrm{HCC}$ recurrence into 2 patterns, those within and those beyond the Milan Criteria. Such a classification would be of great value in assessing the feasibility of further curative resections for patients with $\mathrm{HCC}$ recurrence. It is essential to identify potential markers and factors that are associated with a higher risk of developing recurrent $\mathrm{HCC}$ beyond the Milan Criteria postoperatively. Consequently, more rigorous surveillance and screening strategies (eg, with shorter intervals and more prolonged periods) are necessary for high-risk patients. There may even be a role to study experimental antirecurrence treatment modalities. We appreciate the authors' excellent work. However, we would like to raise the following comments.

First, in this study, a detailed followup program of recurrence surveillance for the included patients was not provided by the authors. We believe that a good recurrence surveillance strategy has a substantial impact on the patterns of recurrence (within or beyond the Milan Criteria). If postoperative recurrence is detected early (within the Milan Criteria), curative therapies such as re-resection, radiofrequency ablation, or liver transplantation are still the possible treatment options for these patients with recurrent HCC. Many hepatic centers recommend 2 to 3 months to be the optimal time interval for postoperative follow-up for patients after curative resection of $\mathrm{HCC}$. In our center, we generally recommend patients to undergo ultrasound and serum alpha fetoprotein once every 1 to 2 months for the first 6 months after resection, then once every 3 months thereafter. This strategy is based on our observation that the early recurrence rate $(<2$ yrs) is much higher than the late recurrence rate $(>=2$ yrs). Unfortunately, not all our patients would stick to this follow-up protocol. Some patients would not come back to see us until they develop symptoms of recurrent tumors. Our experience showed that $\mathrm{HCC}$ recurrence in patients who had regular recurrence surveillance were more likely to have their recurrence detected at an early stage, that is within the Milan Criteria, while those patients who did not have regular follow-up were more likely to have tumor recurrence beyond the Milan Criteria. Hence, the interval of recurrence surveillance is of great significance to the recurrence patterns (within or beyond the Milan Criteria). ${ }^{3,4}$ We failed to find this important information in the reported study.

Second, the influence of the diagnostic tests used in recurrence surveillance on the recurrence patterns would also be important. In general, most centers would use abdominal ultrasound and a serologic alpha-fetoprotein test. For patients with clinically suspected HCC recurrence, cross-sectional images including computed tomography or magnetic resonance imaging would be added. Such tests were neither reported in the study.

As we think, clarification regarding the above-mentioned omissions would greatly consolidate the conclusions of their study.

This work was supported in part by the National Natural Science Foundation of China (Nos. 81472284 and 81672699). interest.

The authors report no conflicts of

Jiongjie Yu, MD

Zheng Wang, MD

Department of Hepatobiliary Surgery

Eastern Hepatobiliary Surgery Hospital, Second Military Medical University, Shanghai, China
Department of Clinical Medicine Second Military Medical University Shanghai, China

Wan Yee Lau, MD, FRCS, FACS, FRACS

(Hon)

Department of Hepatobiliary Surgery

Eastern Hepatobiliary Surgery Hospital, Second Military Medical University,

Shanghai, China

Department of Surgery, Chinese University of Hong Kong, Prince of Wales Hospital Shatin, Hong Kong SAR, China

Tian Yang, MD

Department of Hepatobiliary Surgery Eastern Hepatobiliary Surgery Hospital Second Military Medical University Shanghai, China yangtianehbh@smmu.edu.cn

\section{REFERENCES}

1. Zheng J, Chou JF, Gönen M, et al. Prediction of hepatocellular carcinoma recurrence beyond Milan criteria after resection: validation of a clinical risk score in an international cohort. Ann Surg. 2017;266:693-701.

2. Lee SY, Konstantinidis IT, Eaton AA, et al. Predicting recurrence patterns after resection of hepatocellular cancer. HPB (Oxford). 2014;16:943-953.

3. Arnaoutakis DJ, Mavros MN, Shen F, et al. Recurrence patterns and prognostic factors in patients with hepatocellular carcinoma in noncirrhotic liver: a multi-institutional analysis. Ann Surg Oncol. 2014;21:147-154.

4. Kamiyama T, Nakanishi K, Yokoo H, et al. Recurrence patterns after hepatectomy of hepatocellular carcinoma: implication of Milan criteria utiliz ation. Ann Surg Oncol. 2009;16:1560-1571.

Response to the Comment on "Prediction of Hepatocellular Carcinoma Recurrence Beyond Milan Criteria After Resection: Validation of a Clinical Risk Score in Aninternational Cohort"

$\mathrm{D}$ ear Dr Yu et al,

Our study validated a clinical risk score to predict recurrence following resection of hepatocellular carcinoma (HCC). ${ }^{1}$ Thank you for asking these very important and insightful questions about the impact of surveillance practice on the recurrence pattern following these curative intent resections. The National Comprehensive Cancer Network $(\mathrm{NCCN})$ guideline recommends cross- 
sectional imaging at 3 to 6-month intervals for the first 2 years and then every 6 to 12 months thereafter. ${ }^{2}$ In addition, if patients had elevated preoperative alpha-fetoprotein (AFP), they should be measured every 3 months for 2 years postoperatively, and then every 6 months thereafter. As our study included 5 different institutions spanning North America, Europe, and Asia, the practice may vary slightly depending on insurance coverage under different health care systems, institutional resources, and patients' social factors.

At Memorial Sloan Kettering Cancer Center, we evaluate patients within 2 weeks postoperatively, then follow them every 3 to 4 months in the first 2 years, and then every 6 months thereafter. For surveillance studies, we utilized AFP and liver function tests, and serial computed tomography (CT) scan and/ or magnetic resonance imaging (MRI) as per NCCN guideline. ${ }^{3}$ In addition, we have a Cancer Registry that contacts patients and their providers annually to obtain updated disease status for our prospectively maintained database. During surveillance, when there is a question of recurrence, we review patients' clinical and radiologic information at our weekly Disease Management Team meeting, which are well attended by surgical oncologists, radiologists, medical and radiation oncologists, gastroenterologists, interventional radiologists, and pathologists.

At Washington University at St. Louis and at University of Montreal in Canada, the surveillance practices are as recommended by NCCN. Similarly, at Erasmus Medical Center in the Netherlands, patients were followed postoperatively with CT or MRI combined with serum AFP at 3 to 6 month intervals for up to 2 years, and then followed by annually for up to 5 years. ${ }^{4}$

At Singapore General Hospital and National Cancer Center in Singapore, the first follow-up posthepatectomy is usually in about 2 weeks to review postoperative recovery, and thereafter for HCC surveillance. Patients were followed at intervals of 3 to 6 months for the first 2 years and then at 6 to 12 monthly intervals as per NCCN guideline. Clinical surveillance consisted of clinical evaluations, serial AFP, and hepatic imaging using ultrasound, CT scan, or MRI as deemed appropriate by the surgeon. Of note, as these centers are tertiary referral centers, there are a substantial number of patients who travel from afar for resection and then receive follow-up with their local hepatologists. For example, medical tourism is a major component in Singapore as medical care is better than some of the neighboring Southeast Asian countries. This may affect median length of follow-up or frequency of follow-up, but the median follow-up was 4.5 years among Singaporean cohort.
The impact of surveillance practice on recurrence pattern following resection of $\mathrm{HCC}$ is indeed a crucial question to address and should be further studied. In this study, we did not evaluate this question but it will be an important pursuit to evaluate the impact of the frequency and total length of serial imaging on earlier detection of HCC. However, given what we know about HCC growth kinetics, it would seem unlikely that differences in the timing of imaging studies of a few weeks or even a few months would ultimately change the findings, treatment, or prognosis for most patients. The actual impact will be further investigated. Thank you for your thoughtful comments on this letter to the editor.

This work was supported in part by NIH/NCI P30 CA008748 Cancer Center Support Grant.

W.C.C. is the Founder of Pathfinder Therapeutics and a member of the Advisory Board of Novartis. interest.

The authors report no conflicts of

Jian Zheng, MD

Department of Surgery

Memorial Sloan Kettering Cancer Center New York, NY

William C. Chapman, MD

Department of Surgery

Washington University School of Medicine St. Louis, MO

Simon Turcotte, MD Department of Surgery Université de Montréal, Montreal QC, Canada

Bas Groot Koerkamp, MD, PhD Department of Surgery

Erasmus MC, University Medical Center Rotterdam, The Netherlands

Ser Yee Lee, MD

Department of Hepatopancreatobiliary and Transplant Surgery Singapore General Hospital Singapore, Singapore

Division of Surgical Oncology National Cancer Center Singapore, Singapore

William R. Jarnagin, MD

Department of Surgery

Memorial Sloan Kettering Cancer Center New York, NY jarnagiw@mskcc.org

\section{REFERENCES}

1. Zheng J, Chou JF, Gonen M, et al. Prediction of hepatocellular carcinoma recurrence beyond Milan criteria after resection: validation of a clinical risk score in an international cohort. Ann Surg. 2017;266:693-701.

2. Benson AB 3rd, Abrams TA, Ben-Josef E, et al. NCCN clinical practice guidelines in oncology: hepatobiliary cancers. J Natl Compr Canc Netw. 2009; 7:350-391.

3. Lee SY, Konstantinidis IT, Eaton AA, et al. Predicting recurrence patterns after resection of hepatocellular cancer. HPB (Oxford). 2014;16:943953.

4. Witjes CD, Polak WG, Verhoef C, et al. Increased alpha-fetoprotein serum level is predictive for survival and recurrence of hepatocellular carcinoma in non-cirrhotic livers. Dig Surg. 2012;29:522-528.

\section{OPEN}

Comment on "Does a Combination of Laparoscopic Approach and Full Fast Track Multimodal Management Decrease Postoperative Morbidity?"

\section{To the Editor:}

Ne read with great interest the article by Le'on Maggiori et al. ${ }^{1}$ In order to assess the postoperative outcomes of combination of laparoscopic approach and full fast track multimodal (FFT) management, the authors assigned patients with colorectal cancer into 2 groups: FFT and LFT (limited fast track program), the latter of which was established as a control. No differences in length of stay (LOS) or postoperative morbidity were, however, obtained between the 2 groups in that study.

The fast track (FT) care program, also known as Enhanced Recovery After Surgery (ERAS) program, is a multimodal approach that aims to minimize the physiologic impact of surgery and anesthesia. ${ }^{2}$ As recommended by several guidelines with regard to clinical practice of ERAS protocol in perioperative management, there are 15 to 25 recommended items that might contribute to the improvement of clinical outcome. ${ }^{3-5}$ The relative significance of each element, however, remains unknown because of the lack of definitive support from evidence-based medicine. ${ }^{6}$ Moreover, the complete implementation of all these recommended items, 